Published in final edited form as:

Nat Struct Mol Biol. 2014 October ; 21(10): 876-883. doi:10.1038/nsmb.2878.

\title{
TRIM28 regulates RNA polymerase II promoter proximal pausing and pause release
}

\author{
Heeyoun Bunch ${ }^{1}$, Xiaofeng Zheng ${ }^{2, \S}$, Adam Burkholder ${ }^{3, \S}$, Simon T. Dillon ${ }^{1,4}$, Shmulik \\ Motola $^{5}$, Gabriel Birrane ${ }^{1,6}$, Christopher C. Ebmeier ${ }^{7}$, Stuart Levine ${ }^{5}$, David Fargo ${ }^{3}$, Guang \\ $\mathrm{Hu}^{2, \dagger}$, Dylan J. Taatjes ${ }^{7, \dagger}$, and Stuart K. Calderwood ${ }^{1, *}$ \\ ${ }^{1}$ Department of Radiation Oncology, Beth Israel Deaconess Medical Center, Harvard Medical \\ School, Boston, Massachusetts 02215, USA \\ ${ }^{2}$ Laboratory of Molecular Carcinogenesis, National Institute of Environmental Health Sciences, \\ National Institute of Health, Research Triangle Park, North Carolina 27705, USA \\ ${ }^{3}$ Integrative Bioinformatics, National Institute of Environmental Health Sciences, National \\ Institutes of Health, Research Triangle Park, North Carolina 27705, USA \\ ${ }^{4}$ Genomics and Proteomics Center, Beth Israel Deaconess Medical Center, Harvard Medical \\ School, Boston, Massachusetts 02215, USA \\ ${ }^{5}$ BioMicro Center, Massachusetts Institute of Technology, Cambridge, Massachusetts 02139, \\ USA \\ ${ }^{6}$ Department of Medicine, Beth Israel Deaconess Medical Center, Harvard Medical School, \\ Boston, Massachusetts 02215, USA \\ ${ }^{7}$ Department of Chemistry and Biochemistry, University of Colorado, Boulder, Colorado 80303, \\ USA
}

\section{Summary}

Promoter proximal pausing of RNA polymerase II (Pol II) is a major checkpoint in transcription. An unbiased search for novel human proteins that could regulate paused Pol II at the HSPAlB gene identified TRIM28. In vitro analyses indicated HSF1-dependent attenuation of Pol II pausing upon TRIM28 depletion, whereas in vivo data revealed de novo expression of HSPA1B and other

\footnotetext{
Users may view, print, copy, and download text and data-mine the content in such documents, for the purposes of academic research, subject always to the full Conditions of use:http://www.nature.com/authors/editorial_policies/license.html\#terms

*To whom correspondence should be addressed: scalderw@bidmc.harvard.edu.

†These authors contributed equally to this work.

$\S$ These authors contributed equally to this work.

Accession Code

ChIP-seq genomic data described in this study have been deposited in the Gene Expression Omnibus under accession number GSE48253.

Author Contributions:

$\mathrm{XZ}$ and GH generated WT and TRIM28 KD mES cell extracts for ChIP-seq and ChIP-qPCR. SM and SL processed ChIP-seq. AB, GH, and DF performed Bioinformatics. STD and CE carried out MS. GB constructed TRIM28 plasmids. HB, DJT, and SKC designed the experiments and wrote the manuscript.

Competing Financial Interests

The authors declare no competing financial interests.
} 
known genes regulated by paused Pol II upon TRIM28 knockdown. These results were supported by genome-wide ChIP-sequencing analyses of Pol II occupancy that revealed a global role for TRIM28 in regulating Pol II pausing and pause release. Furthermore, in vivo and in vitro mechanistic studies suggest that transcription-coupled phosphorylation regulates Pol II pause release by TRIM28. Collectively, our findings identify TRIM28 as a novel factor that modulates Pol II pausing and transcriptional elongation at a large number of mammalian genes.

Promoter proximal pausing of RNA polymerase II (Pol II) represents a major checkpoint in transcription. Typically, Pol II enzymes pause at around $+30-100$ relative to the transcriptional start site (TSS) until activating cellular signals induce elongation ${ }^{1}$. Although promoter proximal pausing was discovered over two decades ago, it was initially thought to occur at only a limited set of genes ${ }^{2}$. Recently however, genome-wide analyses such as Chromatin Immuno- Precipitation followed by sequencing (ChIP-seq) and Global Run-On Sequencing (GRO-seq) have shown that promoter-proximal pausing is widespread ${ }^{1,3-7}$. For instance, approximately $30 \%$ of coding genes and over $70 \%$ of developmental or inducible genes harbor Pol II paused at promoter-proximal sites ${ }^{8,9}$. Thus, promoter-proximal pausing is considered a major cellular mechanism to regulate gene expression.

Although the mechanisms of Pol II pausing and pause release are incompletely understood, several transcription factors are known to regulate these processes. DSIF and NELF induce and stabilize pausing ${ }^{10}$ while TFIIS ${ }^{3}$, Myc, and P-TEFb help release Pol II from the pausing site $^{1}$. P- TEFb phosphorylates DSIF, NELF, and the C-terminal domain of Pol II (Pol II CTD), which correlates with pause release ${ }^{11}$. HSP70 is a model gene appropriate for study of promoter- proximal pausing. Especially in Drosophila, Pol II pausing has been extensively studied at $H S P 70$ : Pol II pausing occurs around $+30^{12}$ relative to the TSS and factors such as NELF and DSIF ${ }^{10}$ regulate pausing. However, less is known regarding pausing at human genes including HSP70. Although the fundamental mechanisms of promoter-proximal pausing might be evolutionarily conserved, the degree of conservation is uncertain. For example, HSF1, a master $H S P 70$ activator, is conserved in the DNA binding and trimerization domains but is distinctive in its regulatory and activation domains between Drosophila and human ${ }^{13}$. Spt5 (a subunit of DSIF) knock-down (KD) largely controls pausing, but NelfA KD is less effective in mouse embryonic stem (mES) cells ${ }^{1}$, and NELF KD unexpectedly decreases Pol II density at NELF-regulated genes in humans ${ }^{14}$. NELF is less conserved in eukaryotes, and is lacking in C. elegans and S. cerevisiae. In addition, elongation factors such as Gdown $1^{15}$ and ELL ${ }^{16}$ have been reported to regulate Pol II promoter-proximal pausing in humans yet orthologs for these factors are unknown in Drosophila. These observations imply diversity and complexity of Pol II promoter proximal pause regulatory mechanisms in mammals.

To further probe the factors and mechanisms that regulate Pol II pausing, we initiated an unbiased approach to screen for factors that could selectively bind the non-template strand of the well-studied human HSPA1B (HSP70-2) gene. These experiments were based upon the concept of nucleic acid aptamers, short single-stranded nucleic acid polymers that can bind protein factors with high affinity and selectivity ${ }^{17}$. We reasoned that, for a paused Pol II enzyme, a non- template single-stranded DNA of about 20 bases around the transcription 
bubble could be available for protein factors to bind. Genes regulated by paused Pol II typically contain GC-rich sequences, such as GAGA motifs or the pause button motif, at their promoters ${ }^{18,19}$. The high GC content at the TSS of HSPA1B could facilitate formation of secondary structures that might form a binding motif for a specific protein factor (Supplementary Fig. 1a).

Using single-stranded DNA (ssDNA) oligo-protein binding assays and mass spectrometry, we identified the TRIM28 protein as a factor that bound specifically and selectively to the non- template strand of the HSPA1B promoter. TRIM28 is a multidomain transcriptional regulator linked to activation and repression of a subset of genes ${ }^{20}$. Its abnormal expression is implicated in disorders related to cell growth ${ }^{21}$, development ${ }^{22}$, and differentiation ${ }^{23,24}$. To understand the function of TRIM28 bound at the promoter-proximal pausing site, we performed in vitro transcription assays and in vivo KD experiments. Genome-wide ChIP-seq analyses of Pol II comparing WT and TRIM28 KD mES cells suggest a global function of TRIM28 in controlling Pol II pause release. In addition, a rapid phosphorylation event in TRIM28 was identified upon HSPAIB activation in vivo. We thus propose that a novel pausing factor, TRIM28, regulates Pol II promoter-proximal pausing and progression into processive elongation at a large number of genes in mammals, and regulation of its activity involves post-translational modification.

\section{Results}

\section{TRIM28 binds to the HSPA1B promoter-proximal pausing site}

Firstly, using protein pull-down with the immobilized HSPAIB ssDNA non-template strand, followed by mass spectrometry (MS), we screened for potential unidentified pausing factors. Considering the structural flexibility of non-template ssDNA and its likely accessibility outside the Pol II cleft ${ }^{25}$, we hypothesized that non-template DNA might be recognized by proteins that regulate pausing. The non-template ssDNA oligos of $H S P A 1 B,+1$ to +50 and +1 to +80 along with +1 to +50 of GREB1 (used as a non-pausing control gene; see Supplementary Fig. 1a ${ }^{26}$ were subjected to pull-down assays with HeLa NE. Stringent wash conditions were applied to screen proteins bound to each oligo with high affinity. Only a few proteins were identified with high confidence through such wash conditions by MS (Fig. 1a, Table 1 and Supplementary Fig. 1b). TRIM28 was associated with the +1 to +80 sequence but not with +1 to +50 sequences of HSPAIB or GREBI (Table 1 and Fig. 1b). Immunoblotting confirmed that TRIM 28 specifically bound to the +1 to +80 sequence but not to +1 to +50 of HSPA1B or GREB1 (Fig. 1b). These results suggested that TRIM28 binds to the non-template DNA of HSPAIB, in particular the segment between +50 and +80 . Although TRIM28 is not known as a single- stranded DNA binding protein (e.g. unlike PURb, Fig. 1b), we identified TRIM28 binding motifs, GCCGCG ${ }^{23}$, at +60 to +65 and +62 to +67 of the HSPAIB promoter, both with an $83 \%$ homology to consensus (Fig. 1c). These data prompted further examination to determine whether TRIM28 might function in Pol II promoter-proximal pause regulation. 


\section{Pol II pausing at human HSPA1B in vitro}

A biotinylated fragment of the native human HSPA1B gene $(-467$ to +216$)$ was immobilized onto streptavidin beads. This template was used to assess transcription in vitro using HeLa NE (Fig. 1d,e and Supplementary Fig. 2b). Importantly, our transcription system visualized all species of de novo transcripts, enabling us to monitor transcription activities ranging from abortive initiation to processive elongation. Through this method, a Pol II pausing site was visualized on human $H S P A 1 B$. Upon titrating NE, a stable and prominent RNA transcript was observed at around +70 without detectable run-off or extended transcripts, suggesting a native tendency of the HSPA1B template for Pol II pausing (Fig. 1f). We also noted that Pol II pausing occurred near the TRIM28 binding motif (Fig. 1c). These data suggested that our in vitro system could accurately recapitulate stable Pol II pausing at the $H S P A 1 B$ promoter, with stable pausing occurring at around +70 , near the TRIM28 binding site.

\section{TRIM28 attenuates HSF1-mediated pause release in vitro}

We hypothesized that TRIM28 might repress HSPA1B transcription by augmenting promoter- proximal pausing. To assess this possibility, transcription assays were performed as described (Fig. 1e), using TRIM28-immunodepleted HeLa NE (TRIM28 $\Delta$ NE) (Fig. 2a and Supplementary Fig. 2e). TRIM28 $\Delta$ NE displayed transcription potential comparable to WT NE (Fig. 2b), indicating that TRIM28 depletion does not affect the general transcription efficiency at the $H S P A 1 B$ promoter under these conditions.

In vivo, $\mathrm{HSF} 1$ is rapidly recruited to $H S P 70$ promoters upon heat-shock to activate transcription $^{27}$. In our immobilized template assays, HSF1 enhanced recruitment of PreInitiation Complex (PIC) factors and P-TEFb proteins to the HSPAlB template DNA (Fig. 2c). Therefore, we included HSF1 to recapitulate $H S P A 1 B$ activation in vitro. Native PIC and naturally associating proteins were assembled on the template using NE. Proteins unbound or bound loosely were removed by washing with transcription buffer; NTPs, including radioactively labeled CTP, were then added to initiate transcription and to allow visualization of nascent RNA products (Fig. 1e).

HSF1 was introduced to the transcription reaction at different time points in order to stimulate $H S P A 1 B$ at distinct transcriptional stages: t0, during PIC formation; t1, immediately after PIC formation; and $\mathrm{t} 2$, after Pol II paused at the promoter-proximal site (Fig. 1e). Evidence for Pol II pausing was observed in the absence of HSF1 in both WT and TRIM28 $\Delta$ NE with little evidence of run-off transcripts (Fig. 2d, lanes $1 \& 3$ ). This suggested that Pol II pausing occurred in uninduced HSPA1B with or without TRIM28. When HSF1 was added at t0, transcription was stimulated in both WT and TRIM28 $\Delta$ NEs (Fig. 2d, lanes $2 \& 4$ ), perhaps due to enhanced recruitment of PIC proteins by HSF1 (Fig. 2c). Importantly, pausing was dramatically diminished in TRIM $28 \Delta \mathrm{NE}$, but not in WT NE, when HSF1 was introduced to the established PIC (t1) or the paused complex (t2; Fig. 2e). Thus, HSF1 added at $\mathrm{t} 1$ and $\mathrm{t} 2$ appeared to release paused Pol II more effectively in the absence of TRIM28. Addition of HSF1 at $\mathrm{t} 1$ (after PIC assembly) or $\mathrm{t} 2$ (after formation of paused Pol II) mimics HSF1-mediated HSPAlB activation in vivo in which Pol II has assembled into the PIC or has paused near the promoter. Because paused transcripts $(+70)$ 
were diminished and run-off transcript levels increased in TRIM28 $\triangle \mathrm{NE}$, these results suggested that TRIM28 could be functioning to stabilize paused Pol II in the presence of HSF1.

To verify that other proteins immunodepleted along with TRIM 28 were not contributing to the difference in TRIM28 $\triangle \mathrm{NE}$ activity, we identified proteins bound to the anti-TRIM28 antibody by MS (Fig. 2f and Supplementary Data Source 1,2). Based on their known functions, the proteins that co-immunoprecipitated appeared unlikely to function in promoter-proximal pausing. More importantly, purified TRIM28 re-supplied to TRIM28 $\Delta \mathrm{NE}$ in the transcription assay restored Pol II pausing, validating that TRIM28 was indeed responsible for the formation of stably paused Pol II complexes (Fig. 2g and Supplementary Fig. 2c).

\section{TRIM28 controls expression of known paused genes in vivo}

Because TRIM28 was shown to affect $H S P A 1 B$ transcription in vitro, we next examined whether TRIM28 KD would alter expression of HSPA1B in cells. Since TRIM28 depletion facilitated Pol II pause release in vitro, we hypothesized that Pol II pausing may become attenuated in TRIM $28 \mathrm{KD}$ cells, and increase the basal levels of HSPAlB mRNA expression in vivo. Using two short- hairpin RNA (KD1 and KD2) species targeting TRIM28, the factor was knocked-down in HEK293 cells. As shown in Fig. 3a, the mRNA and protein levels of $H S P A 1 B$, measured by quantitative PCR (qPCR) and immunoblotting, were increased in TRIM28 KD cells but not in controls with scrambled shRNA species. Therefore, as expected, Pol II pausing appeared attenuated in TRIM28 KD cells, permitting Pol II progression from the pause site into the ORF of $H S P A 1 B$.

Recently, it has been reported that genes in immune responsive and signal transduction pathways can be highly paused ${ }^{28}$. Because TRIM28 is known to regulate immune responses and cell proliferation ${ }^{21,24}$, we examined $N F \kappa B$ and $E R K 1$. These genes broadly regulate immune response and proliferation and were shown to have paused Pol II at their promoters ${ }^{28}$. Consistent with this, CDK9 KD reduced the expression of $H S P A 1 B, N F \kappa B$, and $E R K 1$, suggesting these genes are dependent on P-TEFb and thus regulated by Pol II pausing in HEK293 cells (Fig. 3b). We also noted that $N F \kappa B$ and ERK1 possess TRIM28 binding motifs within their promoter regions ( +47 to +52 for $E R K 1$ and +7 to +12 for $N F K B$ with $100 \%$ consensus). Immunoblotting and qPCR indicated up-regulation of $N F \kappa B$ and ERK1 in TRIM28 KD cells (Fig. 3c).

\section{TRIM28 regulates Pol II pausing genome-wide}

Existing ChIP-chip data indicate that TRIM28 is frequently located near the TSS of many protein-coding genes ${ }^{23}$. Based upon our cellular, biochemical, and in vitro data, we hypothesized that TRIM28 may function in Pol II promoter proximal pausing genome-wide. ChIP-seq was therefore performed to investigate the global impact of TRIM28 on Pol II occupancy. Given the overall coding conservation between mouse and human ${ }^{29}$, murine ES $(\mathrm{mES})$ cells provided a useful model system for this investigation. Specifically, the pausing genes evaluated in this study such as EGR1, JUN, ERK1, and HSPAIB are highly conserved between human and mouse with over $92 \%$ coding similarity. 
TSS and TTS-proximal windows were defined as start or end + or -250 respectively. Gene body windows were defined as +500 to +2500 ; if the size of a gene was smaller than 2500 $\mathrm{bp}$, the gene body window was defined as +500 to the gene end. When TRIM28 was knocked-down using siRNA in mES cells (Fig. 4a), the pausing index (Pol II TSS occupancy/gene body occupancy) was either increased or decreased by over twofold at $41.3 \%$ of mappable protein coding genes, compared to WT. As expected, the pausing index of established paused genes such as JUN and EGRl was decreased in TRIM28 KD (Fig. 4b). The pausing index of $H S P A I B$ and $E R K I$ was decreased consistent with the in vivo $\mathrm{KD}$ and gene expression results (Fig. 3 and $4 b$ ).

We also report a series of genes in different signaling pathways whose pausing index was notably altered by TRIM28 KD (Table 2). In agreement with our results, a metagene analysis based on previous TRIM28 ChIP-chip data ${ }^{23}$ indicate that TRIM28 bound genes increased the mean gene body Pol II occupancy upon TRIM28 KD (Fig. 4c and Supplementary Fig. 3a,b). Importantly, TRIM28 KD led to elevation of Pol II occupancy within the gene body (between +250 and +999 from the TSS) at a number of coding genes (Fig. 4d and Supplementary Fig. 3c-e and 4a-d). TRIM28 KD also increased Ser2-phospho Pol II occupancy within the gene body, between +250 and +999 from the TSS, as shown by metagene analysis of 15,917 coding genes (Fig. 4e). Chromosome views of individual loci (IER5 and DQ072391) further illustrate a role for TRIM28 in Pol II progression (Fig. 4f and Supplementary Fig. 6). At IER5, Pol II occupancy increases downstream of TSS and in the gene body as assessed by total Pol II (Fig. 4f, upper panel) and Ser2-phospho Pol II (Fig. 4f, bottom panel) upon TRIM28 KD. Overall, these results support a novel function for TRIM28 in regulating Pol II promoter-proximal pausing and pause release.

\section{Rapid phosphorylation of TRIM28 upon HSPA1B activation}

We next attempted to identify mechanisms through which TRIM28 inhibition of Pol II pause release might be regulated. We initially asked whether TRIM28 was released from the $H S P A 1 B$ promoter upon activation of $H S P A 1 B$ transcription. Thus HEK 293 cells were heatshocked over a time course of $0,0.5,2$, and 5 minutes and TRIM28 occupancy at HSPA1B was monitored by ChIP followed by qPCR with primer sets to amplify the HSPAlB promoter and TSS (Fig. 5a). As reported for Drosophila $H S P 70^{27}$, HSF1 was rapidly recruited to the HSPAIB promoter within 30 seconds of heat-shock (Fig. 5b). Notably, TRIM28 did not dissociate from the TSS of HSPA1B at these time points (Fig. 5c).

TRIM28 is reportedly phosphorylated at S824 under specific circumstances, which has been associated with chromatin structural changes ${ }^{30-32}$. In addition, a study showed that TRIM28 was phosphorylated at S824 when p21 (CDKNIA), a TRIM28 regulated gene, was transcriptionally activated ${ }^{33}$. Therefore, we inspected TRIM28 S824 phosphorylation at $H S P A 1 B$ upon heat-shock induction. HSPA1B expression in HEK293 cells was induced by heat-shock over a time course of 0, 0.5, 2, and 5 minutes, and phospho-TRIM28 (S824) occupancy at $H S P A 1 B$ was quantified using ChIP followed by qPCR. It was observed that TRIM28 became rapidly phosphorylated at S824, an effect apparent within 30 seconds of heat-shock, and dramatically increased in an activating signal dependent manner (Fig. 5d). 
To search for the kinase that phosphorylates TRIM28 at S824, we utilized immunoprecipitation followed by MS. Because protein kinases were not among the few proteins that co- immunoprecipitated with TRIM28 under stringent wash conditions, we initiated a set of TRIM28 IP experiments that utilized less-stringent washes followed by medium salt elutions (Supplementary Fig. 7b). Through this method, DNA-dependent Protein Kinase (DNA-PK) was identified by MS to co-immunoprecipitate with TRIM28 (See Supplementary Data for list of identified proteins). In addition, Ataxia Telangiectasia Mutated (ATM), another member of the PI-3 kinase family, is known to phosphorylate TRIM28 at S824 in the DNA repair process ${ }^{32,33}$. Therefore, we investigated whether DNAPK or ATM were required for TRIM28 phosphorylation at S824 during HSPAlB activation by using specific inhibitors for each kinase, NU7441 ${ }^{34}$ and KU55933 ${ }^{34}$. In addition, an HSF1 inhibitor, KRIBB $11^{35}$ which allows HSF binding to the promoter but interferes with HSF-mediated P-TEFb recruitment, was included to test whether HSF1-P-TEFb interaction was responsible for TRIM28 phosphorylation at S824. Each kinase inhibitor (NU7441, 2 $\mu \mathrm{M}$; KU55933, $10 \mu \mathrm{M}$; KRIBB11, $10 \mu \mathrm{M}$ in $0.1 \%$ DMSO as a final concentration) was added to HEK293 cells an hour prior to 5-minute heat-shock (or non- heat shock control). The results showed that both DNA-PK and ATM inhibitors effectively reduced heat-shock induced TRIM28 S824 phosphorylation at the TSS of HSPA1B upon heat- shock (Fig. 5e). By contrast, the HSF1-P-TEFb inhibitor, KRIBB11 did not affect this phosphorylation, suggesting that TRIM28 S824 phosphorylation is not mediated by P-TEFb. Under similar experimental conditions, we measured Pol II occupancy at the TSS and a distal site of HSPA1B. Total Pol II occupancy at the TSS was increased with or without each of the inhibitors upon heat-shock (Fig. 5e), which implied that these inhibitors did not markedly influence Pol II recruitment to the TSS upon activation.

Importantly, DNA-PK and ATM inhibitors abolished the increase of Pol II occupancy at the $3^{\prime \prime}$ end of $H S P A 1 B$ upon heat-shock, contrasting with DMSO controls, implying reduced Pol II elongation (Fig. 5e). The inhibitor of the HSF1-P-TEFb interaction, KRIBB11, also reduced Pol II occupancy at the 3 " end of HSPA1B, probably due to reduced P-TEFb recruitment. Thus TRIM28 S824 phosphorylation appeared to be regulated by DNA-PK and ATM and correlated with Pol II progression toward the $3^{\prime \prime}$ end and transcriptional elongation at $H S P A 1 B$.

Since a post-translational modification at the C-terminal domain of TRIM28 was involved in release of its repressive function, a C-terminal deletion mutant of TRIM28 (residues 1-617, TRIM28 $\Delta$ CTD, Supplementary Fig. 2c) was tested for its effect on Pol II promoter-proximal

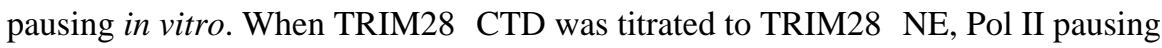
increased in a dose dependent manner upon HSF1 induction (Fig. 5f). This suggested that TRIM28 $\Delta$ CTD could stabilize Pol II pausing.

To further test whether TRIM28 phosphorylation at S824 was critical to regulate Pol II pausing, TRIM28 S824A and S824D mutants were compared with WT TRIM28 in the in vitro transcription assay. Purified TRIM28 (WT, S824A, or S824D) was supplemented with WT or TRIM $28 \triangle$ NE during PIC formation on HSPA1B template DNA (Supplementary Fig. 7a,c). As shown in Fig. 5g,h and Supplementary Fig. 8a,b, WT TRIM28 and the S824A mutant augmented Pol II pausing. By contrast, the TRIM28 S824D mutation (which mimics 
phosphorylation) destabilizes Pol II pausing in vitro. Together with the in vivo data (Fig. 5d,e), these results implicate TRIM28 S824 phosphorylation as a means to regulate Pol II pausing at $H S P A 1 B$. In particular, these data suggest that non-phosphorylated TRIM28 at S824 functions in stabilizing Pol II pausing whereas S824-phosphorylated TRIM28 facilitates Pol II pause release at $H S P A 1 B$.

\section{Discussion}

Using an unbiased approach, we have identified TRIM28 as a novel pausing factor that binds to the non-template DNA near the Pol II pausing site of HSPA1B. Our results suggest that TRIM28 can stabilize paused Pol II and that TRIM28 helps regulate release of paused Pol II complexes. TRIM28 depletion in vitro facilitated Pol II pause release by HSF1. In vivo, TRIM28 contributes to Pol II promoter proximal pausing genome wide, based upon experiments that showed increased Pol II occupancy downstream from the TSS $(>+250)$ at a wide spectrum of genes following TRIM28 knockdown. In addition, we have shown that TRIM28 was phosphorylated at S824 upon HSPA1B activation, dependent on DNA-PK and ATM. Our in vivo and in vitro data suggest that TRIM28 phosphorylation at S824 regulates Pol II pause release.

TRIM28 has been shown to be a powerful suppressor of transcription through the formation of complexes with ZNFs and HP1 proteins ${ }^{36}$, and TRIM28 interacts with the repressive NuRD complex and SETDB $1{ }^{37}$. Previous studies have shown that TRIM 28 phosphorylation at $\mathrm{S} 824$ can promote chromatin relaxation ${ }^{30,32}$; similar chromatin-based changes could facilitate Pol II release from the pausing site of $H S P A 1 B$. It will be of future interest to determine whether this phosphorylation event regulates TRIM28 interaction with histone modifiers such as SETDB1 and the NuRD complex at HSPA1B. Notably, TRIM28 interaction with SETDB1 and the NuRD complex requires sumoylation on TRIM28, and this modification is inhibited by phosphorylation of TRIM28 at S82433,37. Furthermore, nucleosome occupancy is enriched at many genes immediately downstream of the Pol II pausing site at the +1 nucleosome ${ }^{38}$ and TRIM28 may thus function in stabilizing paused Pol II through interaction with such structures.

Our observation that TRIM28 regulates Pol II pausing and pause release at a broad array of protein-coding genes suggests that diverse regulatory mechanisms and signaling pathways might converge upon TRIM28 to regulate Pol II activity. TRIM28 is known to locate at the TSS of genes that regulate the cell cycle and growth, and deregulation of TRIM28 expression is closely linked to development of different cancers ${ }^{23,39}$. Consistently, our Pol II ChIP-seq data indicated that TRIM28 KD alters the pausing index of genes involved in cell growth and apoptotic pathways. It is plausible that disruption of TRIM28 function or expression de-regulates expression of key growth or tumor suppressor genes by altering the activity of paused pol II complexes. Thus, TRIM28 and the signaling pathways that control its phosphorylation state may provide a means to selectively control this key regulatory checkpoint, which could ultimately yield new therapeutic approaches for a subset of cancers. 


\section{Online Methods}

\section{Non-template DNA-Pulldown assay}

Avidin beads were suspended in $0.1 \mathrm{M} \mathrm{KCl}$ HEMG. Forty $\mu \mathrm{l}$ of the beads were incubated with 1.4 nmoles of biotinylated oligos for 30 minutes at room temperature (RT). The oligo amount per reaction was determined by the concentration provided by the manufacturer and also quantified by denaturing PAGE (Fig. 1b). After spin- down, the supernatant was discarded and the beads were incubated with $1.6 \mathrm{mg}$ of the nuclear extracts for 2 hours at 4 ${ }^{\circ} \mathrm{C}$. Unbound proteins were removed by discarding the supernatant. The beads were washed with $0.5 \mathrm{M} \mathrm{KCl} \mathrm{HEMG} \mathrm{(20} \mathrm{mM} \mathrm{HEPES,} \mathrm{pH} \mathrm{7.6,} \mathrm{10 \%} \mathrm{glycerol,} 500 \mathrm{mM} \mathrm{KCl}, 0.1 \mathrm{mM}$ EDTA $7 \mathrm{mM} \mathrm{MgCl}_{2}$ ) including $0.1 \% \mathrm{NP} 40$, followed by a final wash with $0.1 \mathrm{M} \mathrm{KCl}$ HEMG ( $\left.7 \mathrm{mM} \mathrm{MgCl}_{2}, 0.02 \% \mathrm{NP} 40\right)$. After washes, the bound proteins were eluted with the elution buffer (2\% Sarkosyl, 0.02\% NP40, 0.1 M HEMG). The elution was analyzed by the SDS- PAGE followed by silver-staining, MS, and WB.

\section{Mass Spectrometry}

SDS-PAGE gel slices were processed for LC/MS/MS based protein identification using the following method. Gel slices were washed $3 \mathrm{X}$ in $50 \%(\mathrm{v} / \mathrm{v})$ acetonitrile. Proteins in each gel slice were then reduced by incubation in $100 \mathrm{mM} \mathrm{NH}_{4} \mathrm{CO}_{3}, 10 \mathrm{mM}$ DTT at o $56^{\circ} \mathrm{C}$ for 45 minutes. The DTT was washed away and reduced disulfide bonds were blocked by incubation in a solution of $100 \mathrm{mM} \mathrm{NH}_{4} \mathrm{CO}_{3}, 55 \mathrm{mM}$ iodoacetamide (IAA). The gel slice was then washed $3 \mathrm{X}$ to remove the IAA. Proteins were digested by incubating in $100 \mathrm{mM}$ $\mathrm{NH}_{4} \mathrm{CO}_{3}$ containing $0.05 \mathrm{mg} / \mathrm{ml}$ sequencing grade bovine trypsin (ABSciex) overnight at $37^{\circ} \mathrm{C}$. Generated peptides were extracted from gel slices by washing $3 \mathrm{X}$ in $40 \%$ (v/v) acetonitrile, $1 \%$ TFA, then collecting and pooling the supernatant. The volume of extracted peptides was reduced using vacuum centrifugation. Peptides from each gel slice were then run over a reverse phase column (C18 PepMap, Dionex) using nanoflow LC (Ulitmate Plus, Dionex) and printed to 4800 MALDI target plates by an in-line printing robot (Probot, Dionex) that mixed in CHCA $(5.0 \mathrm{mg} / \mathrm{ml}$ stock solution) as the matrix. Peptides were identified by MALDI-TOF/TOF mass spectrometry on an ABSciex 4800 Plus instrument. Protein Pilot 3.0 software was used for peptide and protein identification.

\section{Immobilized template assay and transcription assay}

Dynabeads M-280 Streptavidin (Invitrogen) was prepared with 2X B\&W buffer (10 mM Tris-HCl, $\mathrm{pH} 7.5,1 \mathrm{mM}$ EDTA, $2 \mathrm{M} \mathrm{NaCl}$ ) and incubated with the biotin-conjugated $H S P A 1 B$ template DNA $(-467$ to +216$)$ at $2 \mathrm{fmol} \mathrm{DNA} / \mu \mathrm{g}$ beads. The template-conjugated beads were washed with $1 \mathrm{X}$ B\&W buffer and $0.1 \mathrm{M}$ Buffer D (20\% Glycerol, $20 \mathrm{mM}$ HEPES, pH 7.6, pH 7.9, $0.1 \mathrm{mM}$ EDTA, $100 \mathrm{mM} \mathrm{KCl)}$. Twenty five $\mu \mathrm{g}$ of beads-DNA complex was mixed with TF buffer $\left(12.5 \mathrm{ng} / \mu \mathrm{dII}-\mathrm{dC}, 0.075 \% \mathrm{NP} 40,5 \mathrm{mM} \mathrm{MgCl}_{2}, 250\right.$ $\mathrm{ng} / \mu \mathrm{l}$ BSA, $12.5 \%$ Glycerol, $100 \mathrm{mM} \mathrm{KCl,} 12.5$ mM HEPES, pH 7.6, 62.5 $\mu \mathrm{M}$ EDTA, 10 $\mu \mathrm{M} \mathrm{ZnCl}_{2}$ ) for pre-incubation with transcription factors when indicated as t0. The resultant template-protein complex was pulled-down using a magnet stand (Invitrogen) and resuspended in NE buffer (17.5 ng/ $\mu \mathrm{l} \mathrm{dI}-\mathrm{dC}, 0.1 \% \mathrm{NP} 40,7.5 \mathrm{mM} \mathrm{MgCl}_{2}, 1.25 \mu \mathrm{g} / \mu \mathrm{l} \mathrm{BSA}$, 8.7\% Glycerol, 8.7 mM HEPES, pH 7.6, $44 \mu \mathrm{M}$ EDTA, $130 \mathrm{mM} \mathrm{KCl,} 10 \mu \mathrm{M} \mathrm{ZnCl}_{2}$ ). HeLa $\mathrm{NE}$ was added at $160 \mu \mathrm{g} /$ reaction and incubated for 30 minutes at room temperature (RT). 
The loosely or unbound proteins were removed by multiple washes with Wash buffer (0.0125\% NP40, $833 \mathrm{nM} \mathrm{MgCl2,} \mathrm{12.5 \%} \mathrm{Glycerol,} 12.5 \mathrm{mM}$ HEPES, $\mathrm{pH}$ 7.6, $62.5 \mu \mathrm{M}$ EDTA, $500 \mathrm{mM} \mathrm{KCl}$ ). The proteins stably bound to the template were eluted by $2 \%$ Sarkosyl for Western blotting. For transcription assay, the steps are identical described above until the HeLa NE incubation except that $100 \mathrm{ng}$ of the template DNA and $160 \mu \mathrm{g}$ of HeLa NE per a reaction were used to assemble PIC. The template-protein complex was washed briefly with a 10 beads volume of TW buffer (13 mM HEPES, pH 7.6, 13\% Glycerol, $60 \mathrm{mM} \mathrm{KCl}, 7 \mathrm{mM} \mathrm{MgCl}, 7 \mathrm{mM}$ DTT, $100 \mu \mathrm{M}$ EDTA, 0.0125\% NP40, $10 \mu \mathrm{M}$ $\mathrm{ZnCl}_{2}$ ) and then resuspended in Transcription Buffer I (13 mM HEPES, pH 7.6, $13 \%$ Glycerol, $60 \mathrm{mM} \mathrm{KCl}, 7 \mathrm{mM} \mathrm{MgCl} 2,10 \mu \mathrm{M} \mathrm{ZnCl}_{2}, 7$ mM DTT, $100 \mu \mathrm{M}$ EDTA, $15 \mathrm{ng} / \mu \mathrm{l}$ $\mathrm{dI}-\mathrm{dC}, 10 \mathrm{mM}$ Creatine phosphate). When indicated as $\mathrm{t} 1$, a transcription factor(s) was added immediately after supplying Transcription Buffer I. A mixture of NTP in final concentrations of $250 \mu \mathrm{M} \mathrm{A} / \mathrm{G} / \mathrm{U}$ and $10 \mu \mathrm{M} \mathrm{C}$ and 5-10 $\mu \mathrm{Ci}$ CTP was added to initiate Pol II to polymerize mRNA molecules. When indicated as $\mathrm{t} 2$, a transcription factor(s) was introduced after 10-15 minutes of NTP addition. The reaction was allowed for 30 or 45 minutes as a total incubation time and collapsed with 5 volumes of $1.2 \mathrm{X}$ Stop buffer $(0.6 \mathrm{M}$ Tris-HCl, pH 8.0, $12 \mathrm{mM}$ EDTA, $100 \mu \mathrm{g} / \mathrm{mL}$ tRNA). The mixture was treated with an equal volume of Phenol: Chloroform: Isoamyl alcohol (25:24:1) to extract proteins and then the soluble phase was precipitated with 2.6 volumes of $100 \%$ Ethanol. RNA transcripts were separated in denaturing polyacrylamide gels and exposed to X-ray film. In order to evaluate the transcriptional potential of nuclear extracts or to characterize the HSPA1B template DNA, titrating amounts of WT or TRIM28 $\triangle$ NE between $160 \mathrm{ng}$ and $1.6 \mu \mathrm{g}$ were mixed with $50 \mathrm{ng}$ of the HSPAIB template DNA in Transcription buffer II (13 mM HEPES, pH 7.6, 13\% Glycerol, $60 \mathrm{mM} \mathrm{KCl,} 7 \mathrm{mM} \mathrm{MgCl} 2,1 \mathrm{mM}$ DTT, $100 \mathrm{nM}$ EDTA, $200 \mathrm{ng} / \mu \mathrm{BSA}, 1$ $\mu \mathrm{M} \mathrm{ZnSO}_{4}$ ), followed by incubation for 10 minutes. Transcription was initiated by NTP and allowed for 30 minutes before stopping the reaction and collecting RNA molecules as described above. In the TRIM28 replenishment experiment, approximately $50 \mathrm{ng}$ of purified full-length TRIM28 was added in $160 \mu \mathrm{g}$ of WT or TRIM28 $\Delta \mathrm{NE}$ and incubated for 10-20 minutes at RT before incubating the mixture with the DNA template. The full-length TRIM28 was assayed in $100 \mu \mathrm{M}$ instead of routine $10 \mu \mathrm{M}$ of $\mathrm{ZnCl}_{2}$ in the buffers described above. For the replenishment and titration experiment of TRIM28 $\Delta \mathrm{CTD}, 16 \mu \mathrm{g}$ of NE or TRIM28 $\triangle$ NE pre-supplemented with purified TRIM28 $\triangle \mathrm{CTD}$ was incubated with $50 \mathrm{ng}$ of the HSPAIB template DNA in Transcription buffer II for 10-20 minutes at RT. Then, ATP was added in a $500 \mu \mathrm{M}$ final concentration and the mixture was incubated for 10 minutes before addition of NTP ( $500 \mu \mathrm{M} \mathrm{A}, \mathrm{G}$, and U; $20 \mu \mathrm{M} \mathrm{C}$ in the final concentration) to initiate transcription. HSF1 was added in 10 minutes after NTP. All transcription reactions were done in $25 \mu \mathrm{l}$ in a final volume per a reaction. TF, NE, TW, and Transcription buffer I described above included fresh protease inhibitors of $1 \mathrm{mM}$ Benzamidine, $0.25 \mathrm{mM}$ PMSF, aprotinin, sigma A6279, in 1:1000, and $1 \mathrm{mM}$ Na metabisulfite.

\section{ChIP-seq}

Replicate libraries from input DNA as well as both S2 and total Pol II ChIP-seq from wild type and TRIM28 KD mES cells were examined. Sequence data from all 12 libraries were individually quality filtered using a mean base quality score $<$ Q20 threshold. Filtered reads were then aligned as single end 36 mers to the mouse $\mathrm{mm} 9$ reference genome index using the 
Bowtie short-read alignment program (v0.12.8 employing parameters -v2, -m1) retaining reads mapped to unique genomic locations with at most 2 mismatches. Aligned reads were then de-duplicated using Picard tools for subsequent analyses and the generation of coverage tracks. DNA fragment sizes were estimated to be approximately 150nt using HOMER 4.1 ChIP-Seq analysis tool. Reads were thus center shifted half the fragment length $75 n t$ for downstream analyses. Gene model annotations were downloaded from the mm9 UCSC Known Gene table on March 18th 2013. Genes annotated to chrM as well as random chromosomes were excluded from downstream analyses. Additionally, in order to differentiate TSS-proximal and gene body search windows, genes smaller than 1000nt were excluded. Genes with multiple annotations initiating from the identical TSS were limited to a single entry to minimize redundant search spaces. For comparative analyses, replicates were combined and normalized by uniquely mappable depth, based on the assumption that global PolII occupancy is the same in WT and KD samples. Normalized DNA input reads were then subtracted for each window examined (search windows with negative read counts were set to zero). TSS-proximal windows were defined as start + or - 250; gene body windows were defined as +500 to +2500 or gene end if smaller than 2500 , and the unique mappability of single end 36mers was determined for all search windows. Pausing indexes were defined as the ratio of reads per uniquely mappable base in the TSS-proximal window to the gene body window; gene models in which the TSS-proximal or gene body window was less than $50 \%$ mappable were excluded from pausing index-based analyses. Heat maps were generated using Partek Genomics Suite 6.5 and depict total Pol II ChIP-seq normalized and input-subtracted read counts in $50 \mathrm{nt}$ bins, tiling the -500 to +999 region about each TSS. All heat maps are sorted by WT gene body Pol II occupancy, defined as the total normalized, input-subtracted read count in bins from +250 to +999 . Box plots were generated using R 3.0.1, and depict change in gene body PolII ChIP-seq reads per kilobase. Genes were defined as bound by TRIM28 as in the original publication ${ }^{23}$, while $\mathrm{CpG}$ island intersection was based on the TSS + or -250 region and the UCSC Browser CpG Islands track, downloaded November 2013. Genomic data described in this study have been deposited in the Gene Expression Omnibus under accession number GSE48253.

DNA templates, plasmids, protein purification, and cells and standard methods (Western blot and immunoprecipitation, qPCR, and ChIP) are described in Supplementary Note. Original images of gels, autoradiographs and blots used in this study can be found in Supplementary Data Set 1.

\section{Supplementary Material}

Refer to Web version on PubMed Central for supplementary material.

\section{Acknowledgments}

We appreciate S. Elledge at Harvard Medical School for mediating collaborations and R. Young and D. Orlando at Massachusetts Institute of Technology (MIT) for providing with helpful comments and perspectives for the manuscript. We thank A. York, A. Schubert, M. Knuesel, T. Westerling, and J. Stinchfield for technical support and thank C. Wu in US National Cancer Institute (NCI) for HSF1 vector, R. Kelm Jr. at the University of Vermont for PURb antibody, and F. Zhu at the Florida State University for GST-TRIM28 vectors. We appreciate the Alper, the Pollak, and the Brown labs at Beth Israel Deaconess Medical Center for technical assistance, equipment, and discussions. First author thanks M. Cross and M. A. Stevenson at Beth Israel Deaconess Medical Center for 
administrative supports and J. Park and D. Bunch for loving encouragement throughout the work. This study was supported by grants from US National Institutes of Health (RO-1CA047407) and The Harvard JCRT to SKC and HB, by the Koch Institute for Integrative Cancer Research at MIT (P30-CA14051) and the MIT Center for Environmental Health Sciences (P30-ES002109) to SM and SL, and also by the NCI (R01 CA127364) and the American Cancer Society (RSG 0927401DMC) to DJT. This research was supported in part by the Intramural Research Program of the National Institutes of Health, National Institute of Environmental Health Sciences (1ZIAES102745-02) to GH and XZ and to $\mathrm{AB}$ and $\mathrm{DF}$.

\section{References}

1. Rahl PB, et al. c-Myc regulates transcriptional pause release. Cell. 2010; 141:432-45. [PubMed: 20434984]

2. Rasmussen EB, Lis JT. In vivo transcriptional pausing and cap formation on three Drosophila heat shock genes. Proc Natl Acad Sci U S A. 1993; 90:7923-7. [PubMed: 8367444]

3. Nechaev S, et al. Global analysis of short RNAs reveals widespread promoter- proximal stalling and arrest of Pol II in Drosophila. Science. 2010; 327:335-8. [PubMed: 20007866]

4. Muse GW, et al. RNA polymerase is poised for activation across the genome. Nat Genet. 2007; 39:1507-11. [PubMed: 17994021]

5. Core LJ, Waterfall JJ, Lis JT. Nascent RNA sequencing reveals widespread pausing and divergent initiation at human promoters. Science. 2008; 322:1845-8. [PubMed: 19056941]

6. Zeitlinger J, et al. Whole-genome ChIP-chip analysis of Dorsal, Twist, and Snail suggests integration of diverse patterning processes in the Drosophila embryo. Genes Dev. 2007; 21:385-90. [PubMed: 17322397]

7. Seila AC, et al. Divergent transcription from active promoters. Science. 2008; 322:1849- 51. [PubMed: 19056940]

8. Adelman K, Lis JT. Promoter-proximal pausing of RNA polymerase II: emerging roles in metazoans. Nat Rev Genet. 2012; 13:720-31. [PubMed: 22986266]

9. Gaertner B, et al. Poised RNA polymerase II changes over developmental time and prepares genes for future expression. Cell Rep. 2012; 2:1670-83. [PubMed: 23260668]

10. $\mathrm{Wu} \mathrm{CH}$, et al. NELF and DSIF cause promoter proximal pausing on the hsp70 promoter in Drosophila. Genes Dev. 2003; 17:1402-14. [PubMed: 12782658]

11. Peterlin BM, Price DH. Controlling the elongation phase of transcription with P-TEFb. Mol Cell. 2006; 23:297-305. [PubMed: 16885020]

12. Gilchrist DA, et al. NELF-mediated stalling of Pol II can enhance gene expression by blocking promoter-proximal nucleosome assembly. Genes Dev. 2008; 22:1921-33. [PubMed: 18628398]

13. Rabindran SK, Giorgi G, Clos J, Wu C. Molecular cloning and expression of a human heat shock factor, HSF1. Proc Natl Acad Sci U S A. 1991; 88:6906-10. [PubMed: 1871105]

14. Sun J, Li R. Human negative elongation factor activates transcription and regulates alternative transcription initiation. J Biol Chem. 2010; 285:6443-52. [PubMed: 20028984]

15. Cheng B, et al. Functional association of Gdown1 with RNA polymerase II poised on human genes. Mol Cell. 2012; 45:38-50. [PubMed: 22244331]

16. Byun JS, et al. ELL facilitates RNA polymerase II pause site entry and release. Nat Commun. 2012; 3:633. [PubMed: 22252557]

17. Ulrich H. DNA and RNA aptamers as modulators of protein function. Med Chem. 2005; 1:199_ 208. [PubMed: 16787315]

18. Hendrix DA, Hong JW, Zeitlinger J, Rokhsar DS, Levine MS. Promoter elements associated with RNA Pol II stalling in the Drosophila embryo. Proc Natl Acad Sci U S A. 2008; 105:7762-7. [PubMed: 18505835]

19. Lee $\mathrm{C}$, et al. NELF and GAGA factor are linked to promoter-proximal pausing at many genes in Drosophila. Mol Cell Biol. 2008; 28:3290-300. [PubMed: 18332113]

20. Iyengar S, Ivanov AV, Jin VX, Rauscher FJ 3rd, Farnham PJ. Functional analysis of KAP1 genomic recruitment. Mol Cell Biol. 2011; 31:1833-47. [PubMed: 21343339]

21. Chen L, et al. Tripartite motif containing 28 (Trim28) can regulate cell proliferation by bridging HDAC1/E2F interactions. J Biol Chem. 2012; 287:40106-18. [PubMed: 23060449] 
22. Messerschmidt DM, et al. Trim 28 is required for epigenetic stability during mouse oocyte to embryo transition. Science. 2012; 335:1499-502. [PubMed: 22442485]

23. $\mathrm{Hu} \mathrm{G}$, et al. A genome-wide RNAi screen identifies a new transcriptional module required for selfrenewal. Genes Dev. 2009; 23:837-48. [PubMed: 19339689]

24. Chikuma S, Suita N, Okazaki IM, Shibayama S, Honjo T. TRIM28 prevents autoinflammatory T cell development in vivo. Nat Immunol. 2012; 13:596-603. [PubMed: 22544392]

25. Vassylyev DG, et al. Structural basis for substrate loading in bacterial RNA polymerase. Nature. 2007; 448:163-8. [PubMed: 17581591]

26. Kininis M, Isaacs GD, Core LJ, Hah N, Kraus WL. Postrecruitment regulation of RNA polymerase II directs rapid signaling responses at the promoters of estrogen target genes. Mol Cell Biol. 2009; 29:1123-33. [PubMed: 19103744]

27. Zobeck KL, Buckley MS, Zipfel WR, Lis JT. Recruitment timing and dynamics of transcription factors at the Hsp70 loci in living cells. Mol Cell. 2010; 40:965- 75. [PubMed: 21172661]

28. Gilchrist DA, et al. Regulating the regulators: the pervasive effects of Pol II pausing on stimulusresponsive gene networks. Genes Dev. 2012; 26:933-44. [PubMed: 22549956]

29. Makalowski W, Boguski MS. Evolutionary parameters of the transcribed mammalian genome: an analysis of 2,820 orthologous rodent and human sequences. Proc Natl Acad Sci U S A. 1998; 95:9407-12. [PubMed: 9689093]

30. Ziv Y, et al. Chromatin relaxation in response to DNA double-strand breaks is modulated by a novel ATM- and KAP-1 dependent pathway. Nat Cell Biol. 2006; 8:870-6. [PubMed: 16862143]

31. White D, et al. The ATM substrate KAP1 controls DNA repair in heterochromatin: regulation by HP1 proteins and serine 473/824 phosphorylation. Mol Cancer Res. 2012; 10:401-14. [PubMed: 22205726]

32. Lee DH, et al. Phosphoproteomic analysis reveals that PP4 dephosphorylates KAP- 1 impacting the DNA damage response. EMBO J. 2012; 31:2403-15. [PubMed: 22491012]

33. Li X, et al. Role for KAP1 serine 824 phosphorylation and sumoylation/desumoylation switch in regulating KAP1-mediated transcriptional repression. J Biol Chem. 2007; 282:36177-89. [PubMed: 17942393]

34. Chen BP, et al. Ataxia telangiectasia mutated (ATM) is essential for DNA-PKcs phosphorylations at the Thr-2609 cluster upon DNA double strand break. J Biol Chem. 2007; 282:6582-7. [PubMed: 17189255]

35. Yoon YJ, et al. KRIBB11 inhibits HSP70 synthesis through inhibition of heat shock factor 1 function by impairing the recruitment of positive transcription elongation factor $b$ to the hsp70 promoter. J Biol Chem. 2011; 286:1737-47. [PubMed: 21078672]

36. Wolf D, Goff SP. TRIM28 mediates primer binding site-targeted silencing of murine leukemia virus in embryonic cells. Cell. 2007; 131:46-57. [PubMed: 17923087]

37. Ivanov AV, et al. PHD domain-mediated E3 ligase activity directs intramolecular sumoylation of an adjacent bromodomain required for gene silencing. Mol Cell. 2007; 28:823-37. [PubMed: 18082607]

38. Lee W, et al. A high-resolution atlas of nucleosome occupancy in yeast. Nat Genet. 2007; 39:1235-44. [PubMed: 17873876]

39. Herquel B, et al. Transcription cofactors TRIM24, TRIM28, and TRIM33 associate to form regulatory complexes that suppress murine hepatocellular carcinoma. Proc Natl Acad Sci U S A. 2011; 108:8212-7. [PubMed: 21531907] 


\section{Highlights}

- $\quad$ TRIM28 binds specifically to the non-template DNA of human HSPAlB promoter

- $\quad$ TRIM28 controls Pol II pausing and pause release at HSPA1B

- TRIM28 modulates Pol II promoter-proximal pausing and elongation genomewide

- $\quad$ TRIM28 phosphorylation at S824 releases Pol II pausing and occurs upon HSPAIB transcriptional activation 
a

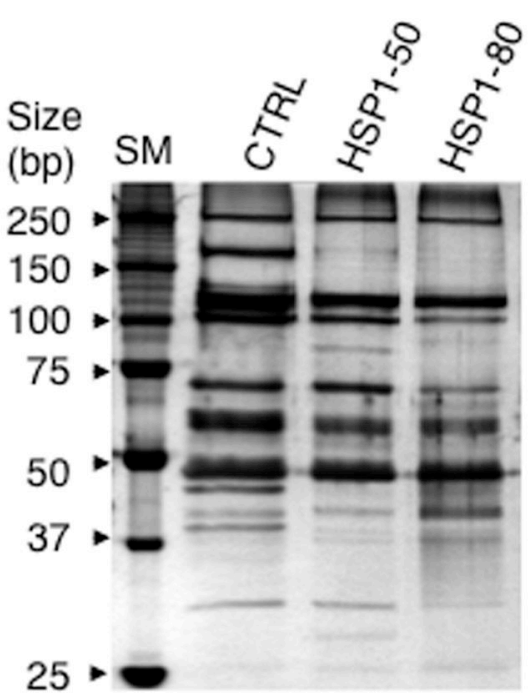

b

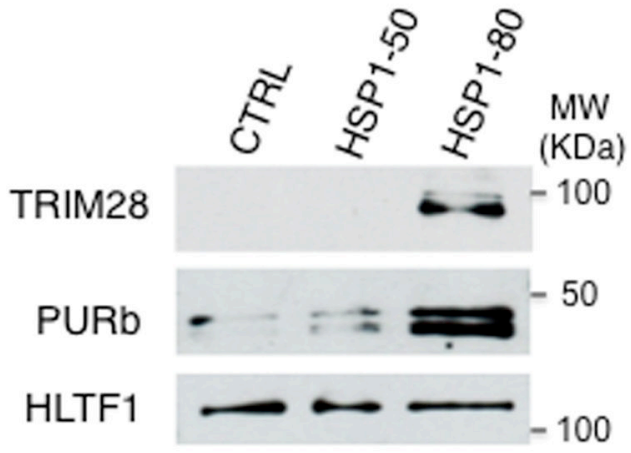

Oligo

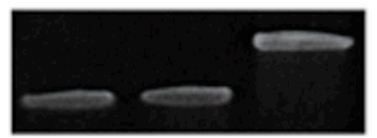

C

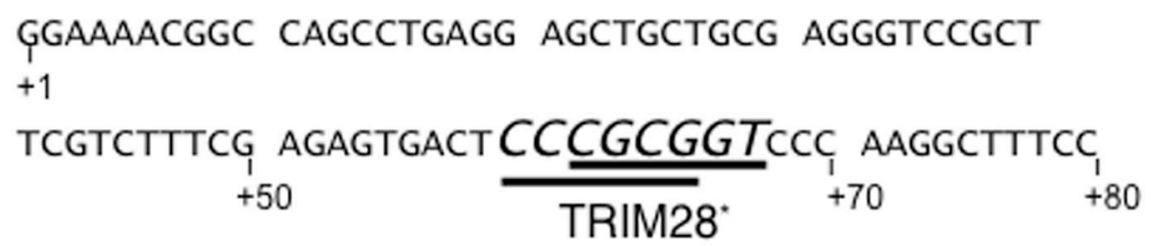

d

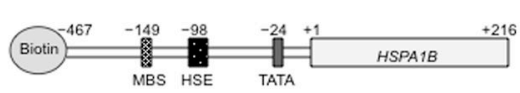

f SM HeLaNE

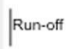

e

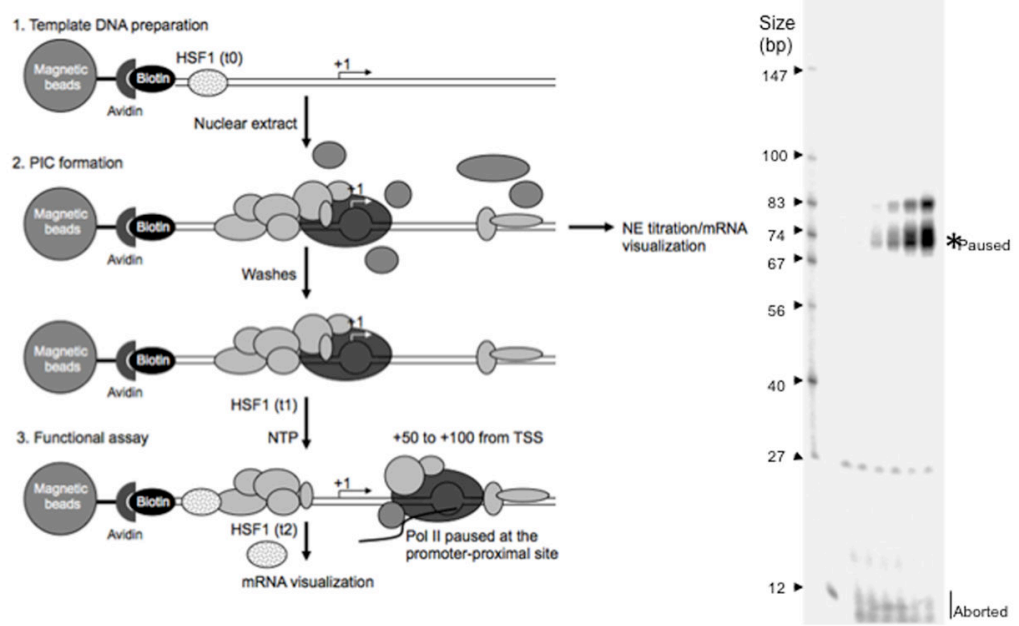

Figure 1. Identification of TRIM28 bound at the HSPA1B promoter-proximal site and in vitro visualization of promoter-proximal pausing in the native human $H S P A 1 B$ gene 
(a) Proteins bound to non-template ssDNAs (silver stain). SM, Size Marker; CTRL, nontemplate (NT) DNA of GREBI +1 to +50; HSP1-50, NT DNA of $H S P A 1 B+1$ to +50 ; HSP1-80, NT DNA of $H S P A 1 B+1$ to +80 . (b) Immunoblots showing specific binding of TRIM 28 to +1 to +80 of the HSPAIB NT DNA. Oligo, the NT DNAs used for the pulldown assay. Uncropped images are shown in Supplementary Data Set 1a (c) The sequence of the HSPA1B promoter- proximal site. TRIM28 Binding Motifs (TRIM28*) are shown in italics and underlined. (d) The immobilized HSPA1B DNA template used in this study. MBS, Myc Binding Site; HSE, Heat Shock Element (HSF1 binding site); TATA, TBP binding motif. (e) A schematic of in vitro transcription assays used in this study. (f) In vitro transcription assay showing a stable promoter- proximal Pol II pause around +70 from TSS of human HSPA1B. SM, Size Marker; Run-off, full- length transcripts; Paused, RNA transcripts generated by paused Pol II; Aborted, short abortive RNA products. 
a

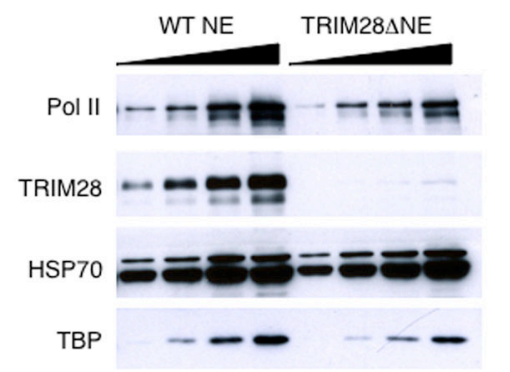

c

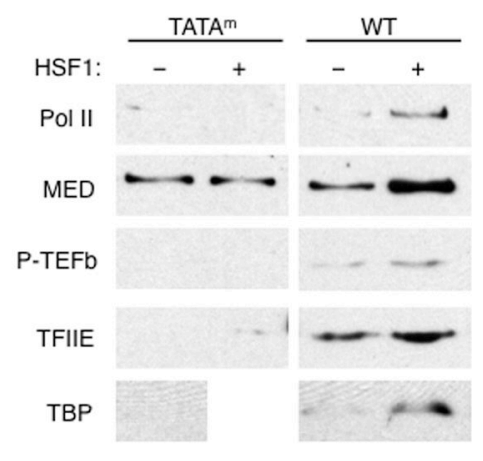

b

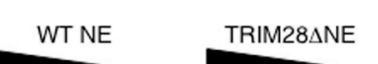

Paused -

TRIM28 $\triangle N E$

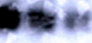

a $=$ as a

d

e HSF1 $\frac{W T}{t 12} \frac{T R I M 28 \Delta}{t 1 t_{2}}$
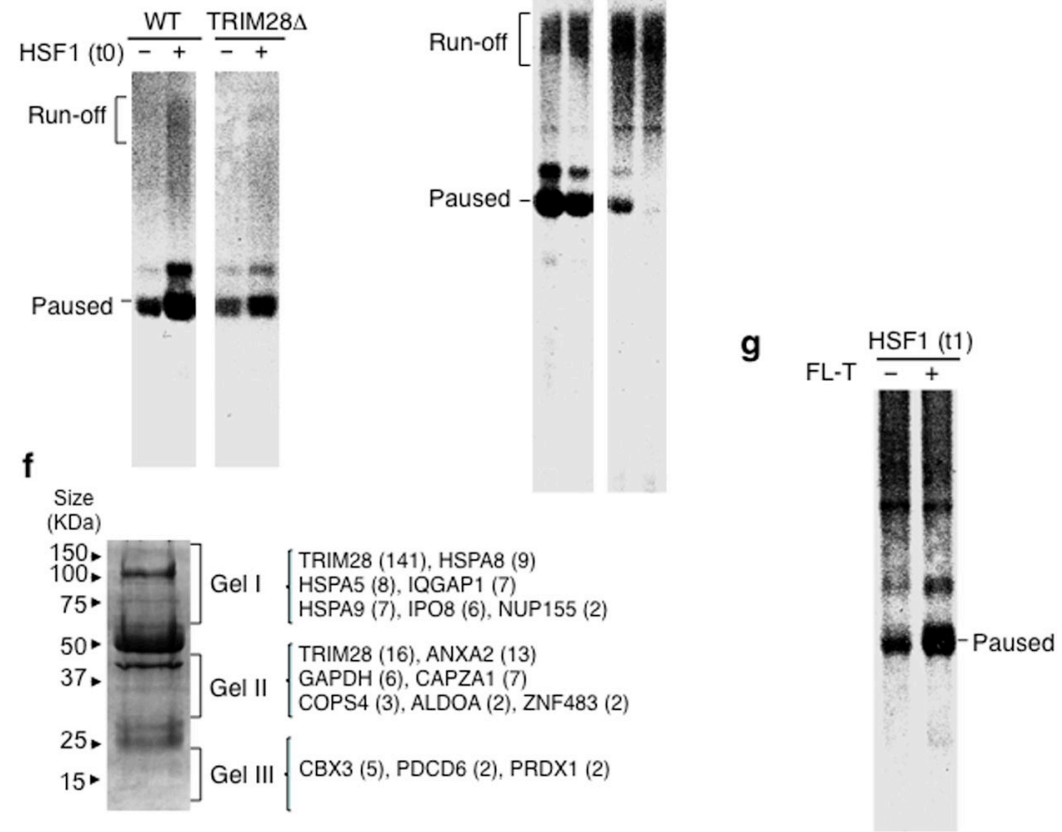

Figure 2. TRIM28 regulates Pol II promoter-proximal pausing upon HSPA1B activation (a) Immunoblots showing TRIM28 immuno-depletion from HeLa NE. Uncropped images are shown in Supplementary Data Set 1b. (b) In vitro transcription assay with the HSPA1B template displaying comparable transcription capability between WT and TRIM28 $\Delta$ NE. An uncropped image is shown in Supplementary Data Set 1c (c) Immobilized template assay showing HSF1 stimulates PIC assembly at HSPA1B. TATA ${ }^{\mathrm{m}}$, TATA box mutant template; WT, wild type template. Uncropped images are shown in Supplementary Data Set 1d. (d) In vitro transcription assay showing HSF1-mediated transcription activation in WT and TRIM28 $\Delta$ NE. t0, HSF1 added during PIC assembly. An uncropped image is shown in Supplementary Data Set 1e. (e) In vitro transcription assay demonstrating the function of TRIM28 in Pol II promoter- proximal pausing. t1, HSF1 supplied to PIC, immediately 
before NTP addition; t2, HSF1 added after Pol II pausing. An uncropped image is shown in Supplementary Data Set 1f. (f) In-gel digestion of gel pieces (Gel I, Gel II, and Gel III) followed by MS identifying the proteins co- immunodepleted with TRIM28. Peptide number identified with each protein is marked in parentheses. (g) In vitro transcription assay showing increased accumulation of paused RNA transcripts with addition of full-length TRIM28 (FL-T) to TRIM28 $\Delta$ NE. An uncropped image is shown in Supplementary Data Set $1 \mathrm{~g}$. 
a
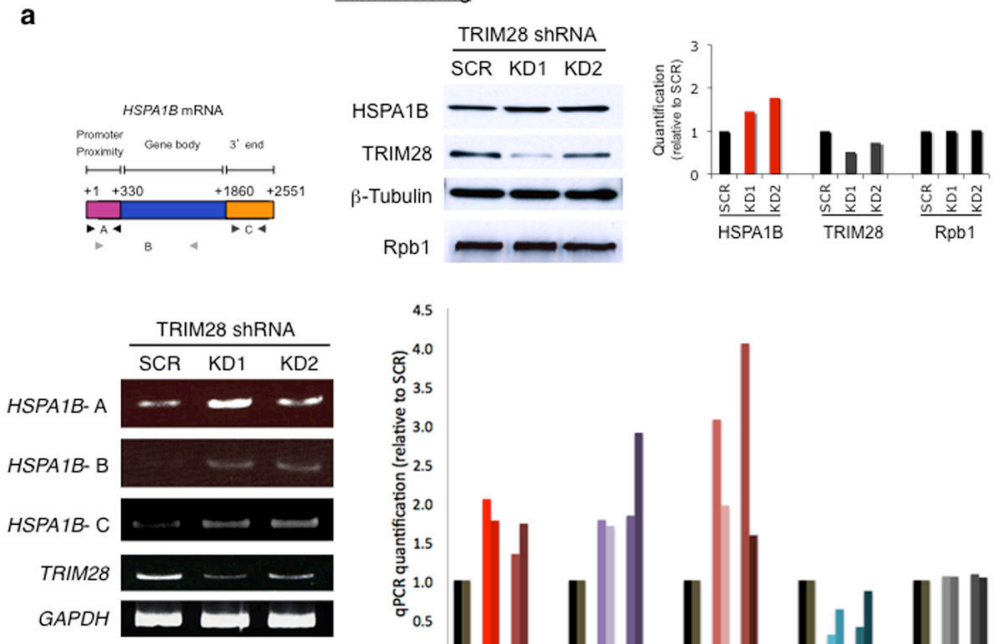

Immunoblotting

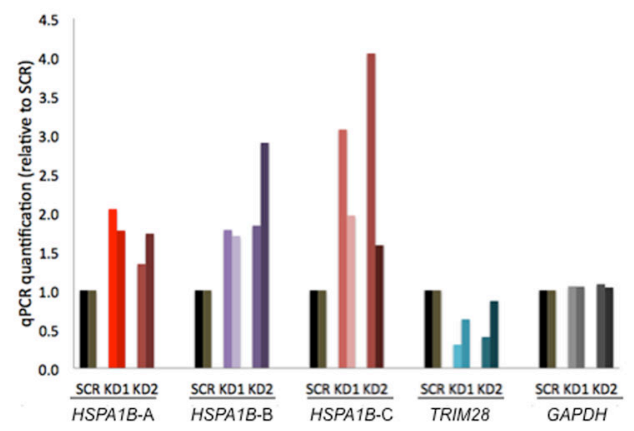

b

Immunoblotting

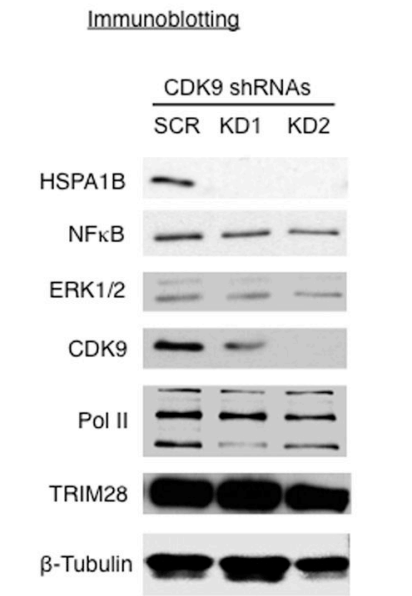

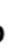

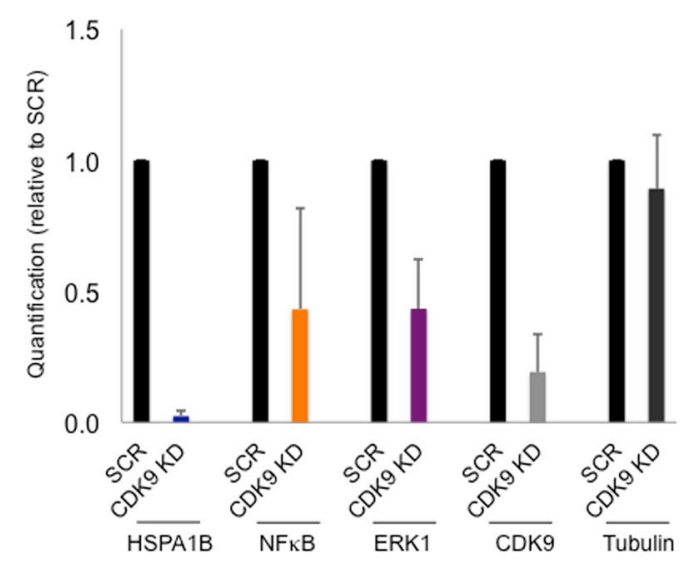




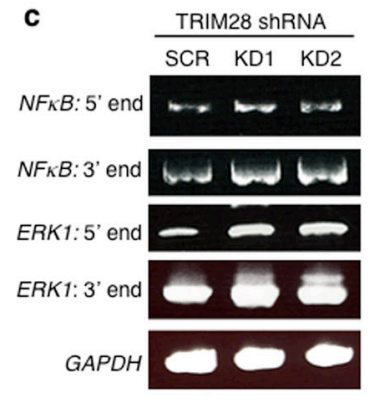

Immunoblotting

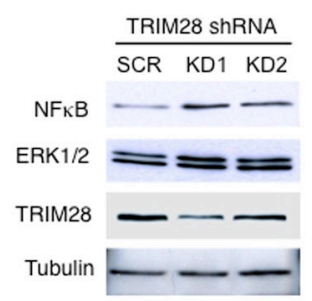

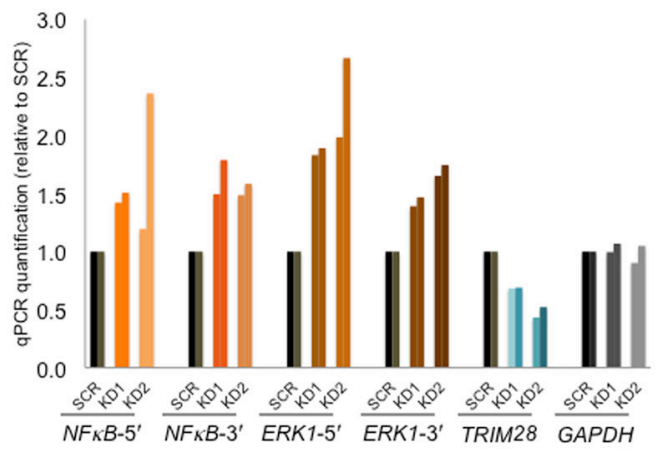

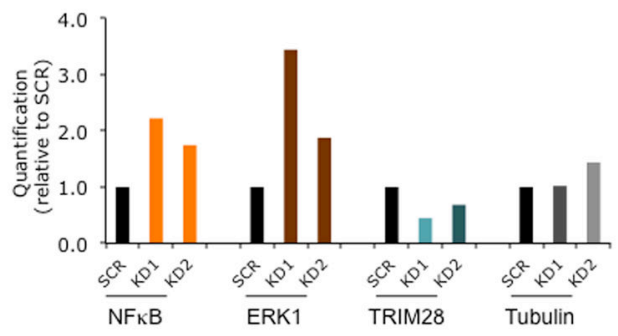

Figure 3. TRIM28 KD increases the expression of paused genes in vivo

(a) HSPA1B mRNA and primer sets for qPCR (arrows, upper left). qPCR data (left and bottom right panel, $n=2$ cell cultures) and western blot (upper right panel) showing increased expression of HSPA1B in TRIM28 KD cells. SCR, HEK293 cells transfected with a scrambled shRNA; KD1 and KD2, TRIM28 KD cells with two different shRNA species targeting TRIM28. Uncropped images are shown in Supplementary Data Set 1h,i,k. (b) Western blot data in CDK9 KD HEK293 cells. SCR, HEK293 cells transfected with a scrambled shRNA; KD1 and KD2, CDK9 KD cells by two different shRNAs. Error bars represent standard deviations ( $\mathrm{n}=3$ cell cultures) (c) $\mathrm{qPCR}(\mathrm{n}=2$ cell cultures) and immunoblotting of $N F \kappa b$ and $E R K 1$, showing increased mRNA (left and upper right panel) and protein (bottom right panel) levels upon TRIM28 KD. Uncropped images are shown in Supplementary Data Set $1 \mathrm{j}, \mathrm{k}$. 
a
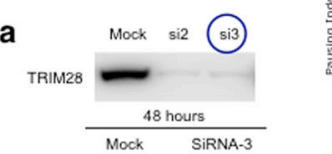

mES

scale bars: $50 \mu \mathrm{M}$

C

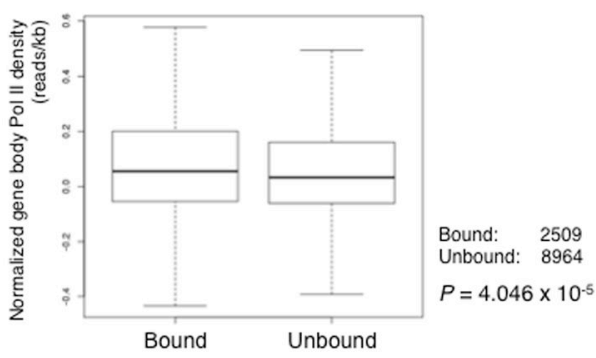

d

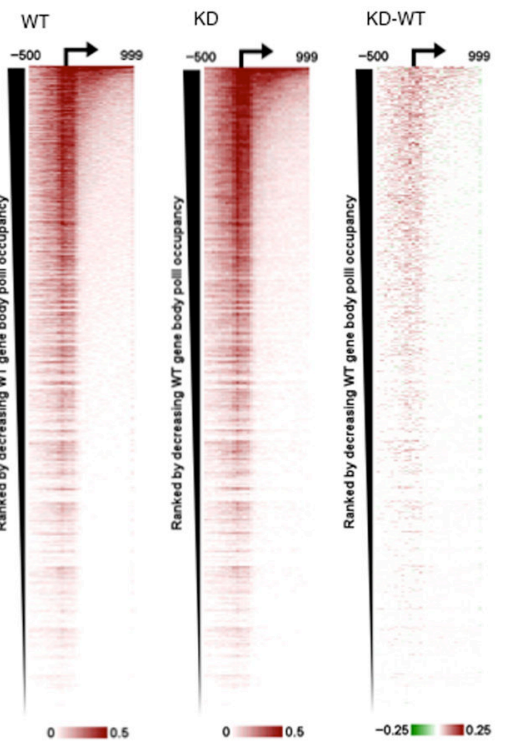

$\mathbf{f}$
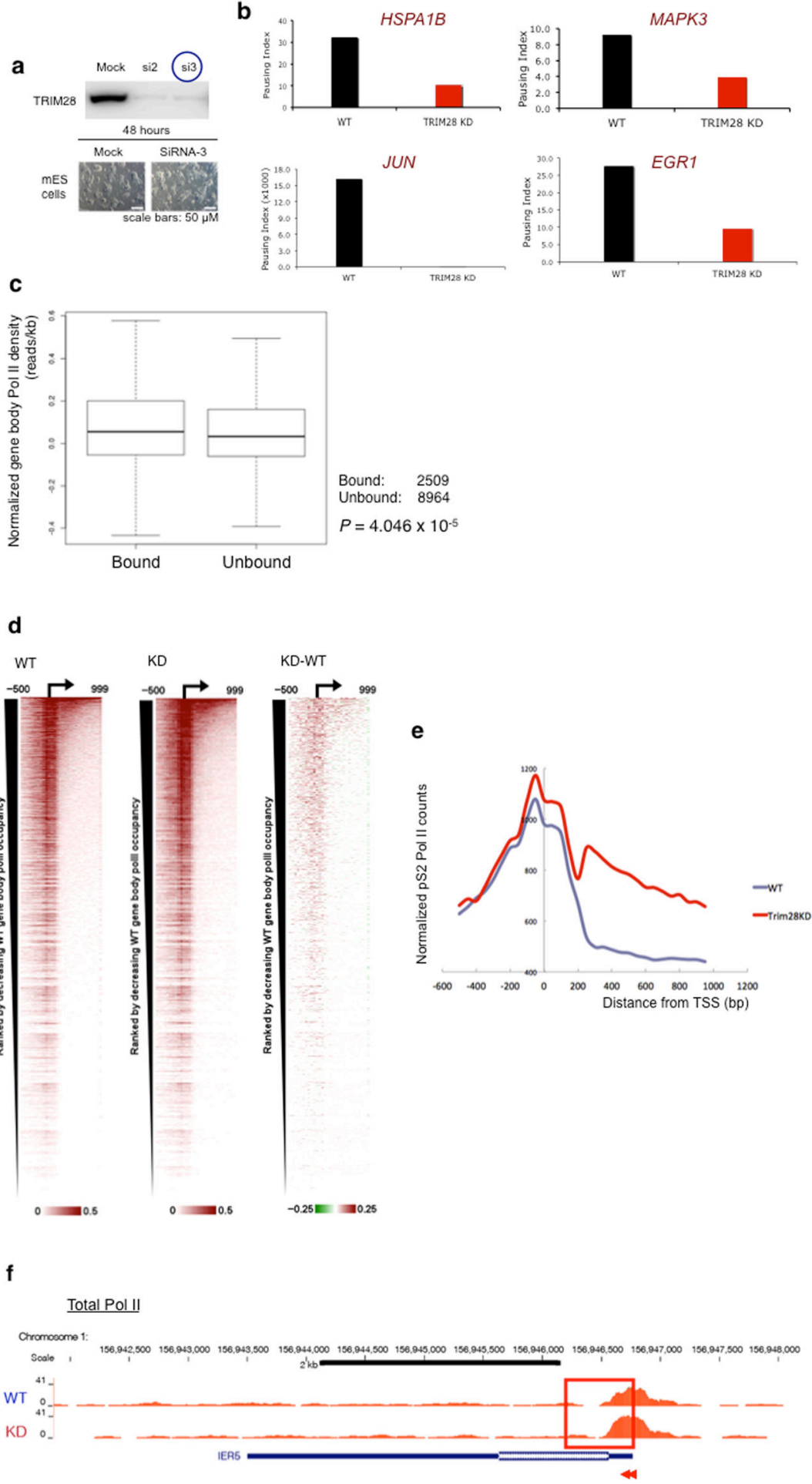

S2Poلl

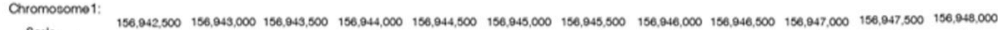

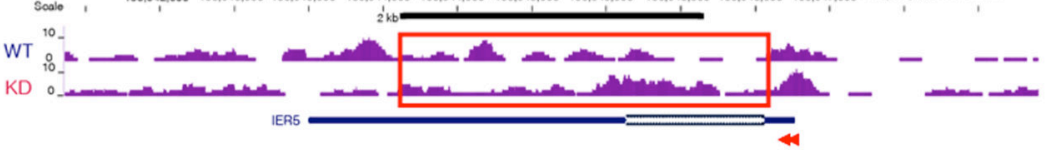

Nat Struct Mol Biol. Author manuscript; available in PMC 2015 April 01. 
Figure 4. TRIM28 regulates Pol II pause release

(a) TRIM28 KD in mES cells using two different siRNAs (si2 and si3). Immunoblotting (upper panel) and cell morphology (bottom panel) are shown, compared with control (Mock). si3 was used for ChIP-seq. (b) Pausing index change at individual genes upon TRIM28 KD. (c) Box plots showing change in gene body Pol II occupancy at paused genes, indicating a modest increase in gene body Pol II for TRIM28 bound genes upon TRIM28 KD (p-value based on Wilcoxon Rank Sum test). Center line: median; Box limits: 25th and 75th percentile (interquartile range); Upper Whisker: largest observation less than or equal to 75 th percentile plus $1.5 \mathrm{x}$ the interquartile range; Lower Whisker: smallest observation greater than or equal to 25 th percentile minus $1.5 \mathrm{x}$ the interquartile range. (d) Heat maps of total Pol II sorted by WT gene body Pol II occupancy (N=32,729). Normalized, input adjusted total Pol II ChIP-seq reads from WT mES cells (WT) and TRIM28 KD (KD), and changes upon knock down (KD-WT) are shown. (e) Metagene analysis from Ser2 phosphoPol II ChIP-seq comparing 15,917 coding genes. (f) Chromosome views of total Pol II and Ser2-Phospho-Pol II (S2 Pol II) in IER5 (Immediate Early Response 5), illustrating facilitated Pol II entry into the downstream of the promoter-proximal pausing site in TRIM28 KD. TSS and orientation of IER5 are marked with arrowheads and the regions with noticeable increase in Pol II occupancy in KD are embraced in red boxes. 
a

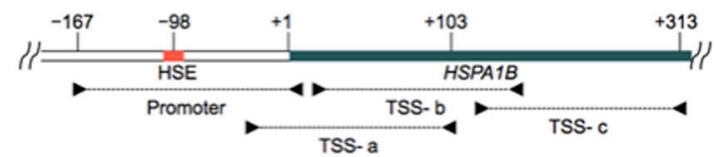

b

C $\underline{\text { Total TRIM } 28}$

d Phospho-TRIM28 (S824)
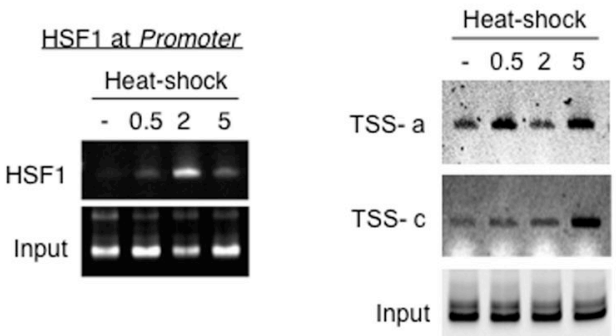

Heat-shock (min) - 0.525

TSS- a

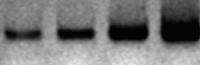

Input

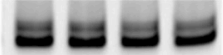

e

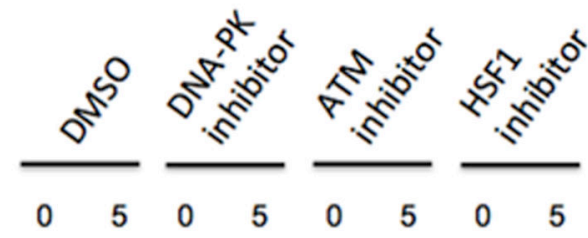

Phospho-TRIM28 (S824) at TSS- $c$

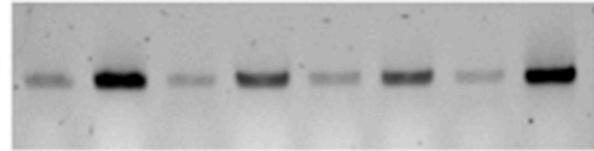

Total Pol II at the 3 ' end $(+1861$ to +2010$)$

Total Pol II at TSS- $c$

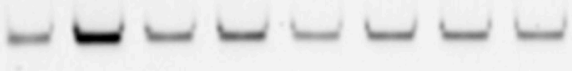

Input

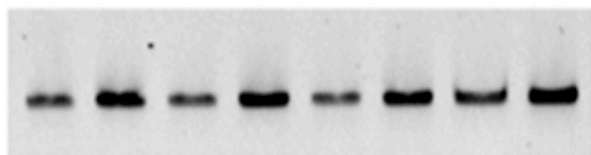




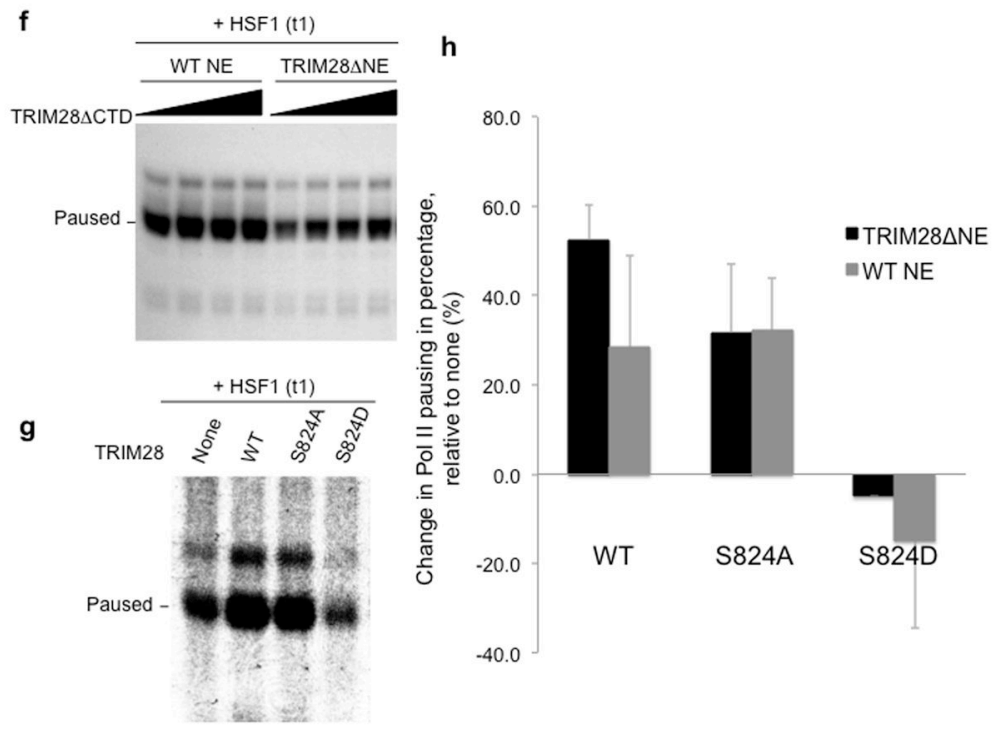

i
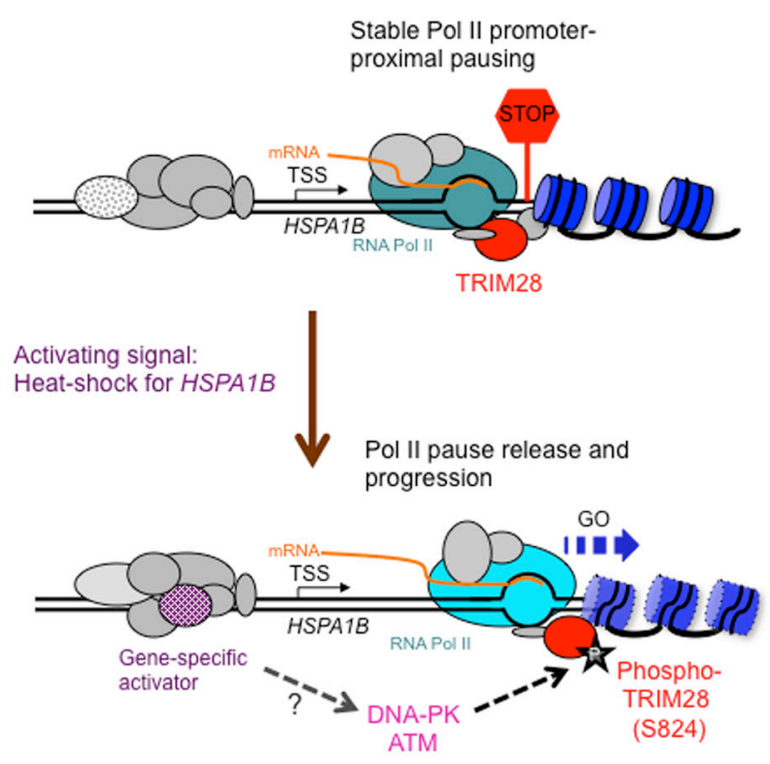

Figure 5. Release of TRIM28-mediated transcriptional repression involves TRIM28 phosphorylation at $\mathbf{8 2 4}$

(a) HSPA1B promoter and TSS with marked primers (arrowheads) used in ChIP-qPCR; HSE, Heat Shock Element (HSF1 binding site). (b) ChIP-qPCR showing rapid association of HSF1 with the HSPA1B promoter upon heat-shock. (c) ChIP-qPCR showing TRIM28 remaining at HSPAIB TSS upon transcriptional activation. (d) ChIP-qPCR showing TRIM28 (S824) phosphorylation upon transcriptional activation. (e) ChIP-qPCR displaying that DNA-PK and ATM inhibitors interfere TRIM28 S824 phosphorylation and Pol II progression into the HSPA1B gene body upon heat-shock. Uncropped images for Fig. 5b-e are shown in Supplementary Data Set 11,m. (f) In vitro transcription assay showing that TRIM28 $\triangle$ CTD omitting S824 stabilizes Pol II pausing. (g) In vitro transcription assay validating that phosphorylation of TRIM28 S824 regulates Pol II pause release at HSPA1B. 
An uncropped image is shown in Supplementary Fig. 8b. (h) The efficiency of WT, S824A or S824D TRIM28 in stabilizing Pol II pausing in vitro was quantified (error bars, s.d., $\mathrm{n}=3$ technical replicates). (i) Model of TRIM28-mediated pausing regulation. Top: TRIM28 bound near the TSS represses transcriptional elongation by stabilizing Pol II promoterproximal pausing. Bottom: a gene-activating signal modulates TRIM28 to suppress its repressive function and Pol II is released from the pausing site into the gene body. At $H S P A 1 B$, TRIM28 remains at the TSS but is phosphorylated at S824 by DNA-PK and ATM upon heat-shock. This post-translational modification correlates with Pol II pause release at HSPAIB. 


\section{Table 1 \\ Summary of MS data from the ssDNA pull-down assay}

Proteins commonly identified from the control (GREB1) and HSPAlB oligos (HSPAlB 1-50 and HSPAlB 1-80) are marked in italics. Seven proteins in bold were confirmed through immunoblotting. The MOWSE score and number of peptide matches are listed in a parenthesis.

\begin{tabular}{c|c|c}
\hline Control (GREB1) & HSPA1B 1-50 & HSPA1B 1-80 \\
\hline CSDA (374;13) & CSDA (616;23) & CSDA (422;21) \\
YBX1 (262;7) & YBXI (601;23) & TRIM28 (232;2) \\
U2AF2 (72;1) & HLTF (93;4) & YBXI (193;12) \\
MSH6 (58;1) & MSH2 (73;3) & PURb (112;2) \\
FEN1 (53;1) & ACACA (71;1) & \\
& PCMT1 (63;2) & \\
& SSBP (62;1) & \\
& PC4 (52;1) & \\
& DHX36 (50;1) & \\
\hline
\end{tabular}

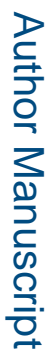

Nat Struct Mol Biol. Author manuscript; available in PMC 2015 April 01. 
Table 2

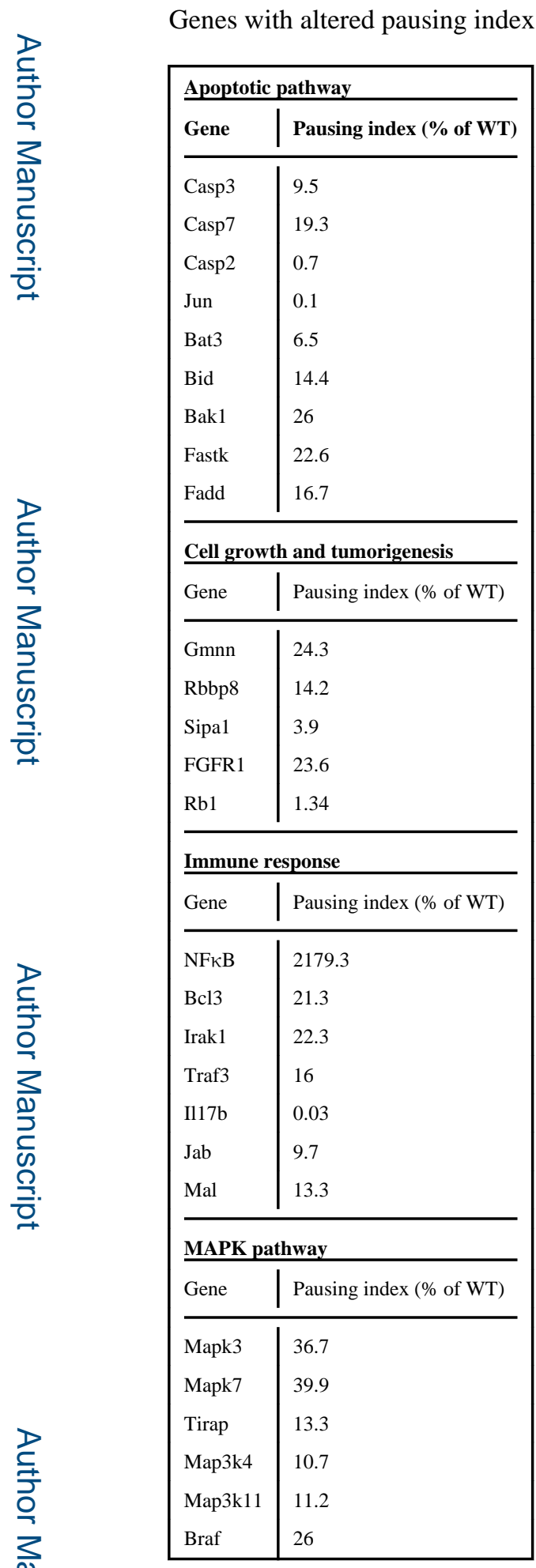

